\title{
Advances in biotechnology: Human genome editing, artificial intelligence and the Fourth Industrial Revolution - the law and ethics should not lag behind
}

Advances in biotechnology have led to human genome editing and to progress in artificial intelligence (Al), fuelling the Fourth Industrial Revolution. With these advances and their gaining momentum come promises for wellbeing at a level not previously imagined. Undoubtedly, along with these promises, ethical, legal and social considerations surface, and valid concerns that the law and ethics are lagging behind justifiably emerge.

Genome editing involves precise additions, deletions and alterations to the genome. Basic science research on genome editing is already underway in laboratories globally. Clinical applications involving somatic (non-reproductive) cells are in the early stages of development, and there is huge potential for the use of this technology in germline (reproductive) cells. ${ }^{[1]}$ Pillay and Thaldar, ${ }^{[2]}$ in this issue, discuss the legal situation, and underscore the point that gene-editing technologies such as the most recent CRISPR system pose challenges to South African (SA) biotechnology law. They highlight the ambiguities within section 57 of the National Health Act $(\mathrm{NHA})^{[3]}$ with regard to the broad definition of reproductive cloning, which possibly includes germline genome editing. Reproductive cloning, in terms of the NHA, is banned, and depending on how its definition is interpreted, the ban could extend to include germline genome editing.

Ethical considerations arise across the spectrum with regard to genome editing - at the levels of basic science research, somatic-cell editing and germline editing. Basic science research is undeniably critical to advancing biomedical science. The same applies to basic science research involving genome editing. Our understanding of the molecular processes that control disease development and progression is advanced by genome-editing research using somatic cells. The potential for this to enable the development of better interventions for people who are affected by these diseases is immense. Genomeediting research of germline cells will assist with the understanding of human development and fertility, and hence could support progress in fertility treatments, regenerative therapies and other related medical applications. ${ }^{[1]}$ Gene therapy, where genetic changes are made to somatic cells, is an established modality of treatment, and genome editing for somatic applications would not be dissimilar. Somatic genome editing could be performed either outside, or directly inside, the body. While the latter could pose technical challenges, in that the gene-editing tools may not find their target genes efficiently, or may inadvertently affect germline cells, clinical trials using this technology for some diseases have already been embarked upon in some countries. Prenatal and preimplantation genetic diagnosis to avert disease transmission has been used for some time already. However, these technologies do not work in some cases, and where they do work, could result in discarding affected embryos, or in selective abortion, giving rise to beginning-of-life age-old debates. Germline genome editing could provide some families with the most appropriate option for averting disease transmission, and the resulting genetic changes would then be passed down the generations. This shift away from purely individual-level effects is viewed as contentious by some. Social and ethical concerns, including those involving the acceptance of children with disabilities, the risk of inheriting off-target genome effects, equitable access and slippery-slope cautions in the contexts of enhancement and eugenics, are being debated. Genome editing for enhancement purposes would involve both somatic and germline processes. Enriching traits and capacities beyond levels considered adequate for health is a realistic possibility, and invokes considerations of fairness, social norms and the need for both public debate and regulation. ${ }^{[1,4]}$

Following broad consultation, the US National Academy of Sciences recommended that heritable genome-editing clinical trials be permitted within a framework of due care and responsible science, which entails that the following criteria must be satisfied:[1]

- an absence of reasonable alternatives;

- restriction to preventing a serious disease or condition;

- restriction to editing genes that have been convincingly demonstrated to cause or to strongly predispose to the disease or condition;

- restriction to converting such genes to versions that are prevalent in the population and are known to be associated with ordinary health, with little or no evidence of adverse effects;

- availability of credible preclinical and/or clinical data on risks and potential health benefits of the procedures;

- ongoing, rigorous oversight during clinical trials of the effects of the procedure on the health and safety of the research participants;

- comprehensive plans for long-term, multigenerational follow-up that still respects personal autonomy;

- maximum transparency, consistent with patient privacy;

- continued reassessment of both health and societal benefits and risks, with broad ongoing participation and input by the public; and

- reliable oversight mechanisms to prevent extension to uses other than preventing a serious disease or condition.

Seven principles for the governance of human genome editing are proposed by the Academy: promoting wellbeing, transparency, due care, responsible science, respect for persons, fairness and transnational co-operation. ${ }^{[1]}$ The Nuffield Council on the ethical acceptability of genome editing in the context of reproduction, has proposed two principles to be satisfied. Firstly, the intention of the intervention is to secure the welfare of the individual born as a result of such technology. Moreover, the intervention must also be consistent with the welfare of such a person. Secondly, principles of social justice and solidarity are upheld, and the intervention should not result in an intensifying of social divides or marginalising of disadvantaged groups in society. ${ }^{[4]}$

Mahomed, ${ }^{[5]}$ in her article in this issue on artificial intelligence (AI) and the Fourth Industrial Revolution in healthcare, highlights the promise of these advances, even in the context of resource scarcity. She briefly addresses the impact and importance of $\mathrm{Al}$ in the healthcare setting, 
and discusses some concerns with regard to the ethical, social and legal challenges that could arise with its implementation in the African context. Just as gene editing, under the umbrella of gene therapy, is not new, Al, too, is not a new notion, and has been recognised in the field of medicine since as far back as the 1970s. What is new is the rapid growth of Al, resulting in the powerful push behind the Fourth Industrial Revolution. Ethical considerations include those around eroding the human element in healthcare, data security and bias, and quality control and standards. ${ }^{[5]}$ The ethical principles recommended by the National Academy and the Nuffield Council, albeit for genome editing, will apply in the context of $\mathrm{Al}$ as well. In addition, some laws may require amendments, and there may be a need for the development of new laws to address possible legal hiatuses in this context.

SA is internationally renowned for its expertise in research. In addition, it has, over the past few decades, developed robust international collaborations in the context of health research. With these advances in science and technology gaining momentum, it is inevitable that both local and international institutions and laboratories will proceed to utilise our local expertise. Academic discussion and debate, coupled with public engagement, are necessary so that ethical and social considerations can be used to inform the updating of our laws. This could allow for suitable safeguards that facilitate ethical research and clinical management, rather than blindly obstruct these advances, to the detriment of our wellbeing. In addition, because laws differ between national jurisdictions, there needs to be mutual respect for differing national policies when international collaborative research is conducted.

\section{Ames Dhai}

Editor

ames.dhai@wits.ac.za

SAfr J Bioethics Law 2018;11(2):58-59. DOI: 10.7196/SAJBL.2018.v11i2.667

1. National Academies of Sciences, Engineering, and Medicine. Human Genome Editing: Science, Ethics and Governance. Washington: National Academies Press, 2017. http://nap.edu/24623 (accessed 20 October 2018).

2. Pillay S, Thaldar D. CRISPR: Challenges to South African biotechnology law. S Afr J Bioethics Law 2018;11(2):90-92. https://doi.org/10.7196/SAJBL.2018.v11i2.653

3. South Africa. National Health Act No. 61 of 2003. GG 26595. https://www.gov.za/ sites/default/files/a61-03.pdf (accessed 2 November 2018).

4. Nuffield Council on Bioethics. Genome editing and human reproduction: Social and ethical issues. Nuffield Council on Bioethics, 2018. http://nuffieldbioethics.org/ project/genome-editing-human-reproduction (accessed 2 November 2018).

5. Mahomed S. Healthcare, artificial intelligence and the Fourth Industrial Revolution Ethical, social and legal considerations. S Afr J Bioethics Law 2018;11(2):93-95 https://doi.org/10.7196/SAJBL.2018.v11i2.664 